\title{
Association of Left Coronary Dominance with In- Hospital Adverse Outcomes after Percutaneous Coronary Intervention in Patients with Acute Coronary Syndrome
}

\author{
Muhammad Azmol Hossain, S T M Abu Azam, Md. Khalequzzaman, Tariq Ahmed Chowdhury, \\ Abul Hasnat Md. Jafar, Sharadindu Shekhar Roy \\ Department of Cardiology, National Institute of Cardiovascular Diseases, Dhaka
}

\begin{abstract}
Key words: Acute coronary syndrome, Percutaneous coronary intervention, Coronary dominance, $M A C E$.

Background: Coronary dominance affects on in-hospital outcomes of patients with acute coronary syndrome and also affects the outcome following percutaneous coronary intervention. Left dominant anatomy is believed to be associated with worse prognoses for patients with acute coronary syndrome undergoing percutaneous coronary. This study evaluated the manner in which coronary dominance affects in-hospital adverse outcomes of acute coronary syndrome (ACS) patients who underwent percutaneous coronary intervention (PCI).

Methods: Data were analyzed from 149 ACS patients who underwent PCI between November 2014 and October 2015 at National Institute of Cardiovascular Diseases (NICVD), Dhaka. The patients were grouped based on diagnostic coronary angiograms performed prior to PCI; those with right dominant plus co-dominant anatomy ( $R D+C o$ group) and those with left dominant anatomy (LD group).

Results: Total adverse in-hospital outcome is $8.7 \%$ patients. In LD group $23.1 \%$ patients were experienced adverse in-hospital outcome, on the contrary $5.7 \%$ of the patients with RD+Co group did have such experience. About 2.7\% patients developed arrhythmia, 2.7\% cardiogenic shock, $2 \%$ acute left ventricular failure and $0.7 \%$ ischaemic chest pain of the both groups. Among them arrhythmia, acute left ventricular failure and cardiogenic shock were more common in LD group than $R D+C o$ (7.7\% vs. $1.6 \%, 7.7 \%$ vs. $0.8 \%$ and $7.7 \%$ vs. $1.6 \%)$ group. Multivariate logistic regression analysis revealed that smoking, diabetes mellitus and left coronary dominance were the independent predictors for developing adverse in-hospital outcome with ORs being 1.317, 1.074 and 6.553 respectively ( $p$ $<0.05)$.

Conclusion: Patients of left coronary dominant had higher in-hospital adverse outcome compared with patients of right dominant plus co-dominant in a population with acute coronary syndrome who underwent percutaneous coronary intervention and left dominant anatomy was an independent predictor for developing adverse in-hospital outcome.
\end{abstract}

(Cardiovasc.j. 2017; 9(2): 129-134)

\section{Introduction:}

Cardiovascular disease (CVD) is the leading cause of mortality worldwide, responsible for one-third of all deaths. According to world health organization (WHO) estimates, 17 million people died of CVD in 2004. Developing countries contributed $80 \%$ of CVD deaths. ${ }^{1}$ This figure is expected to grow to 23.6 million by 2030. Ischaemic heart disease alone caused 7 million deaths worldwide in 2010, an increase of $35 \%$ since 1990 . Commencing in 1980 , the Global Burden of Disease Study has provided the most-comprehensive estimates of disease burden for 235 causes of death and the disease burden attributable to 67 different risk factors in 21 regions of the world. ${ }^{2}$ Coronary artery disease (CAD) is an important medical and public health issue because it is common and leading cause of death throughout the world. Bangladesh has been experiencing epidemiological transition from communicable disease to non-communicable disease (NCD). The overall mortality rate has decreased significantly over the last couple of decades. Of all South Asian countries, Bangladesh probably has the highest rates of CVD and yet is the least studied. ${ }^{3}$

Address of Correspondence: Dr. Muhammad Azmol Hossain, Department of Cardiology, National Institute of Cardiovascular Diseases, Dhaka, Bangladesh. Email:azmolhossain30@gmail.com. 
The use of percutaneous coronary intervention to treat ischemic coronary artery disease (CAD) has expanded remarkably over the past three decades. In the absence of left main or complex multi-vessel CAD, PCI is the preferred method of revascularization. ${ }^{4}$ Percutaneous coronary intervention (PCI) has a considerable evidence base and it is firmly established as the most common procedure used in the invasive treatment of patients with CHD. ${ }^{5}$

Variations in the balance of the coronary arteries are common, particularly with regard to the supply of the posterior aspect of the left ventricle. In the majority of patients, the right coronary artery (RCA) reaches the crux of the heart and supplies the posterior descending artery (PDA). Leftdominant (LD) anatomy has a prevalence of approximately $5-12 \%$ in the general population. In these individuals, the left circumflex artery (LCX) reaches the crux and supplies the posterior descending and, usually, the atrioventricular nodal branches. ${ }^{6}$

Left and codominance are generally considered to be normal variants with no particular prognostic significance. However, the relatively low prevalence of left and codominance may reflect a small biologic disadvantage relative to right dominance. It is possible that left and codominance may rep-resent less well-balanced circulation with more myocardium at risk. This may be particularly true in patients with acute coronary syndrome (ACS) because of culprit lesions in the LCX and left main (LM) territories with either left-dominant or codominant systems. ${ }^{7}$

\section{Methods:}

This prospective observational study was conducted in the National Institute of Cardiovascular Diseases (NICVD), Dhaka from November 2014 to October 2015. Patients with acute coronary syndrome who underwent coronary intervention during the study period were included in the study. Patients with chronic kidney diseases (serum creatinine level $>2 \mathrm{mg} / \mathrm{dl}$ ), chronic liver disease, chronic obstructive pulmonary disease, valvular heart disease, congenital heart disease and cardiomyopathy were excluded. Informed written consent was taken from each patient before enrollment. Meticulous history was taken and detailed clinical examination was performed and recorded in predesigned structured proforma. Patient's data including age, sex, previous history of anginal chest pain, smoking, hypertension (HTN), diabetes mellitus (DM), dyslipidaemia, family history of CAD and clinical findings on admission were taken.

Results of routine investigations such as blood sugar, serum creatinine, serum electrolytes, fasting Lipid profile, Troponin-I, and echocardiography were done. Previous ECG was analyzed and 12 lead resting ECG was done immediately after PCI and next day morning after PCI. Where there was ongoing ischaemic chest pain blood samples were taken 24 hours after procedure for further troponin I to identify post PCI MI.

Coronary angiography was done for patients of acute coronary syndrome during index hospitalization and coronary dominance was detected. PCI was done after coronary angiography.

Patient was divided into two groups according to coronary dominancy. Group I: patient with right plus co-dominant and group II: patient with left dominant. Following PCI, patient was monitored for at least 24 hours and then in ward till discharge or death. Following in-hospital outcomes were observed after percutaneous coronary intervention: a) Ischaemic chest pain with or without new ECG change b) Heart failure c) Cardiogenic shock d) Significant arrhythmia (VT, VF, SVT, AF, CHB, Mobitz type II heart block) \& e) In-hospital mortality.

\section{Results:}

A total of 149 patients with acute coronary syndrome were enrolled in this study. 123 patients were taken with right plus co-dominant (Group I) and 26 patients were taken with left dominant (Group II). The mean age was found $51.46 \pm 10.35$ years with $\mathrm{RD}+\mathrm{Co}$ group and $51.65 \pm 8.98$ years with LD group. Mean age of both group were $51.49 \pm 10.09$ years. Male patient was predominant in the whole study population.

This study found that the most common risk factor was smoking (70.5\%), followed by hypertension (37.6\%), diabetes mellitus (33.6\%), family history of coronary artery disease $(28.2 \%)$ and dyslipidemia (12.1\%). This study found that UA, NSTEMI, STEMI in $\mathrm{RD}+\mathrm{Co}$ group were $21.1 \%, 17.9 \%, 61 \%$ and in $\mathrm{LD}$ group were $7.7 \%, 23.1 \%, 69.2 \%$. According to diagnosis 
of ACS patients, there were found no statistical significant difference between the two groups $(p=0.272)$. According to involvement of vessels and use of type of the stent, there was no statistical significant difference between the two groups.

Total adverse hospital outcome is $8.7 \%$ patients. In group II $23.1 \%$ patients were experienced adverse in-hospital outcome, on the contrary 5.7\% of the patients with group I did have such experience $(p=0.004)$.

In-hospital outcome, total $2.7 \%$ patients developed arrhythmia and it was the most common complication, followed by cardiogenic shock (2.7\%), acute left ventricular failure (2\%) and Ischaemic chest pain (0.7\%) of the both groups. Among them arrhythmia, acute left ventricular failure and cardiogenic shock were more common in LD group than $\mathrm{RD}+\mathrm{Co}(7.7 \%$ vs. $1.6 \%, 7.7 \%$ vs. $0.8 \%$ and $7.7 \%$ vs $1.6 \%$ group. There was one death in patients with RD+Co group.

Multivariate logistic regression analysis revealed smoking, diabetes mellitus and left coronary dominance were independent predictors for developing adverse in-hospital outcome.

Table-I

Baseline clinical characteristics of study population ( $n=149)$.

\begin{tabular}{lccc}
\hline & Group I (n =123) & Group II (n =26) & p Value \\
\hline Age in years (Mean \pm SD) & $51.46 \pm 10.35$ & $51.65 \pm 8.98$ & 0.931 \\
Smoker & $72.4 \%(89)$ & $61.5 \%(16)$ & 0.193 \\
Hypertensive & $35 \%(43)$ & $50 \%(13)$ & 0.113 \\
Diabetics & $35.8 \%(44)$ & $23.1 \%(6)$ & 0.154 \\
Dyslipidemia & $69.9 \%(86)$ & $65.3 \%(17)$ & 0.173 \\
Family history of premature CAD & $26.8 \%(33)$ & $34.6 \%(9)$ & 0.282 \\
LVEF in \% (Mean \pm SD) & $54.37 \pm 8.70$ & $49.11 \pm 4.60$ & 0.003 \\
Hospital stay(days)(Mean \pm SD) & $3.14 \pm 0.53$ & $3.73 \pm 0.72$ & 0.006 \\
LAD & $31.7 \%(39)$ & $23.1 \%(6)$ & \\
RCA & $44.7 \%(55)$ & $42.3 \%(11)$ & 0.061 \\
LCX & $3.3 \%(4)$ & $11.5 \%(5)$ & \\
UA & $21.1 \%(26)$ & $7.7 \%(2)$ & \\
NSTEMI & $17.9 \%(22)$ & $23.1 \%(6)$ & 0.272 \\
STEMI & $61.0 \%(75)$ & $69.2 \%(18)$ & \\
Drug eluting stent (DES) & $53.7 \%(66)$ & $73.1 \%(19)$ & 0.070 \\
Bare metal stent (BMS) & $39.0 \%(48)$ & $15.4 \%(4)$ & \\
Both (DES \& BMS) & $7.3 \%(9)$ & $11.5 \%(3)$ & \\
\hline
\end{tabular}

LVEF - Left ventricular ejection fraction, LAD- Left anterior descending artery, RCA- Right coronary artery, LCX- Left circumflex artery, UA- Unstable angina, NSTEMI- Non ST elevation MI, STEMI- ST elevation MI.

Table-II

In-hospital outcomes in study population $(n=149)$.

\begin{tabular}{lccc}
\hline & Group I $(\mathrm{n}=123))$ & Group II (n = 26) & p Value \\
\hline Ischaemic chest pain & $0.8 \%(1)$ & $0.0 \%(0)$ & 0.645 \\
Arrhythmias & $1.6 \%(2)$ & $7.7 \%(2)$ & 0.141 \\
Acute left ventricular failure & $0.8 \%(1)$ & $7.7 \%(2)$ & 0.023 \\
Cardiogenic shock & $1.6 \%(2)$ & $7.7 \%(2)$ & 0.141 \\
Death & $0.8 \%(1)$ & $0.0 \%(0)$ & 0.826 \\
\hline
\end{tabular}


Table-III

Multivariate logistic regression analysis on in-hospital mortality.

\begin{tabular}{lccc}
\hline Variables of interest & \multicolumn{2}{c}{ Multivariate analysis } & \\
\cline { 2 - 3 } & OR & $95 \%$ CI of OR & p value \\
\hline age $(\geq 50$ years $)$ & 0.839 & $0.246-2.856$ & 0.778 \\
Smoking & 1.317 & $0.339-5.119$ & 0.045 \\
Diabetes mellitus & 1.074 & $0.546-7.876$ & 0.047 \\
Hypertension & 0.900 & $0.267-3.035$ & 0.866 \\
Troponin I & 0.993 & $0.980-1.006$ & 0.295 \\
LVEF $(<50 \%)$ & 0.295 & $0.207-4.329$ & 0.674 \\
Left dominance & 6.553 & $1.984-21.643$ & 0.002 \\
\hline
\end{tabular}

\section{Discussion:}

The mean age was found $51.46 \pm 10.35$ years with right dominant plus co-dominant $(\mathrm{RD}+\mathrm{Co})$ group and $51.65 \pm 8.98$ years with left dominant (LD) group. Mean age of both group were $51.49 \pm 10.09$ years. One of the study in Bangladesh found that mean age was $50.15 \pm 8.8$ years of patients of IHD $^{8}$. Male patient was predominant in the whole study population. About 139 (93.3\%) patients of the study were male and $10(6.7 \%)$ patients were female. Male were 114 (92.7\%) in $\mathrm{RD}+\mathrm{Co}$ group and 25 (96.2\%) in LD group. Hence, the sex distribution of the study patients was almost similar in both groups $(p=0.52)$. Over all male and female ratios was 13.9:1. In another study in Bangladesh male female ratio was 7.01:1. ${ }^{8}$ Female are less prone to developed IHD in premenopausal age due to protective role of estrogen, moreover smoking as a risk factor of IHD is less common in our country among female, which may explain male predominance of IHD.

This study found that the most common risk factor was smoking, followed by dyslipidemia, hypertension, diabetes mellitus and family history of coronary artery disease. All of the risk factors between the two groups were found no statistical significant difference. One of study in Bangladesh found that smoking (81\%) was the highest risk factor, followed by hypertension $34 \%$, diabetes mellitus $32 \%$, family history of CAD $20 \%$ and dyslipidemia $9 \% .{ }^{9}$ This study found that UA, NSTEMI, STEMI in RD+Co group were $21.1 \%$, $17.9 \%, 61 \%$ and in $\mathrm{LD}$ group were $7.7 \%, 23.1 \%$, $69.2 \%$. According to diagnosis of ACS patients, there were found no statistical significant difference between the two groups. One of the study in abroad showed that diagnosis of ACS patients between the $\mathrm{RD}+\mathrm{Co}$ and LD group had no statistically significant difference. ${ }^{6}$

Normal weight had found more in LD group (50\%) than RD + Co group (30.1\%). On the other hand, overweight had found more in $\mathrm{RD}+$ Co group (63.4\%) than LD group (46.2\%). Obesity had also more in RD group (4.9\%) than LD group (3.8\%). The mean BMI was $24.91 \pm 2.65$ and $23.58 \pm 2.11$ in patients of RD + Co group and patients of LD group respectively which was statistically insignificant $(p=0.197)$. Mean BMI of IHD patients of another study ${ }^{10}$ in Bangladesh was $24.1 \pm 4.1$, which was very similar to present study. All the patients were evaluated echocardiographically to see the LV ejection fraction. Mild left ventricular systolic dysfunction more in $\mathrm{LD}$ (73.1\%) group than $\mathrm{RD}+\mathrm{Co}$ (42.3\%) group. Mean ejection fraction was $54.37 \pm 8.70 \%$ in patients with $\mathrm{RD}+\mathrm{Co}$ group and $49.11 \pm 4.60 \%$ in patients with LD group. Statistically significant difference in ejection fraction was found among the study patients $(\mathrm{p}=0.001)$.

This study found that LAD, RCA, LCX, in RD+Co group were $31.7 \%$. 44.7\%, 3.3\% and in $\mathrm{LD}$ group were $23.1 \%, 42.3 \%, 11.5 \%$. According to involvement of vessels, there were found no statistical significant difference between the two groups $(\mathrm{p}=0.061)$. DES was used more in $\mathrm{LD}$ group than RD+Co group but BMS was used more in $\mathrm{RD}+\mathrm{Co}$ group than $\mathrm{LD}$. According to use of type of the stent, there were found no statistical significant difference between the two groups $(p=0.07)$. One 
of the study was also found that DES was used more in $\mathrm{LD}(55.8 \%)$ than $\mathrm{RD}+\mathrm{Co}(54 \%)$ group and BMS was used more in $\mathrm{RD}+\mathrm{Co}(33.9 \%)$ than $\mathrm{LD}$ (30.8\%) group. ${ }^{6}$ It was observed that the mean duration of hospital stay after PCI was $3.14 \pm 0.53$ days in patients with $\mathrm{RD}+\mathrm{Co}$ group and $3.73 \pm 0.72$ days in patients with LD Group. The mean difference was significantly different $(p=0.006)$.

In-hospital outcome, total $2.7 \%$ patients developed arrhythmia, followed by cardiogenic shock (2.7\%), acute left ventricular failure (2\%) and Ischaemic chest pain $(0.7 \%)$ of the both groups. Among them arrhythmia, acute left ventricular failure and cardiogenic shock were more common in LD group than $\mathrm{RD}+\mathrm{Co}$ group. One of the study in abroad showed that left ventricular failure was more (18.8\%) in LD group than $\mathrm{RD}+\mathrm{Co}(14.7 \%)$ group and cardiogenic shock was more (10.3\%) in LD group than $\mathrm{RD}+\mathrm{Co}(7.3 \%)$ group. ${ }^{6}$ There was one death in patients with $\mathrm{RD}+\mathrm{Co}$ group. This patient was dead due to early stent thrombosis.

Total adverse hospital outcome is $8.7 \%$ patients. In LD group $23.1 \%$ patients were experienced adverse in-hospital outcome, on the contrary 5.7\% of the patients with $\mathrm{RD}+\mathrm{Co}$ group did have such experience. So, in-hospital outcome significantly more adverse was $\mathrm{LD}$ group than $\mathrm{RD}+\mathrm{Co}$ group $(p=0.004)$. One of the study was found that adverse in-hospital outcome was more (15.1\%) in LD group than $\mathrm{RD}+\mathrm{Co}$ group (13.6\%). ${ }^{6}$ The higher in-hospital adverse outcome associated with LD patients due to RCA serves as a back-up supply in patients with RD anatomy, providing a measure of protection for the myocardium in ACS patients. Lower left ventricular ejection fractions and larger infarct size is another reason affecting inhospital adverse outcome for LD patients. Multivariate logistic regression analysis was done among traditional predictors of adverse in-hospital outcome such as advanced age (e"50 years), smoking, diabetes mellitus, hypertension, left ventricular ejection fraction and left coronary dominance. Among these, smoking, diabetes mellitus and left coronary dominance were found to be the independent predictor for developing adverse in-hospital outcome with ORs being $1.317,1.074$ and 6.553. respectively and $p$ value $<0.05$.

\section{Conclusions:}

Patients of left coronary dominant had higher inhospital adverse outcome compared with patients of right plus co-dominant in a population with acute coronary syndrome underwent percutaneous coronary intervention.

\section{Study limitations}

This was a non-randomized study. Number of study population was small. It was a single centered study.

\section{Recommendations}

Patients of left dominant anatomy are high risk group in acute coronary syndrome patient undergoing coronary intervention than the right plus co-dominant anatomy. Left dominancy is independent factor of adverse in-hospital outcome in patients with acute coronary syndrome after percutaneous coronary intervention. Caution should be taken in a patient of acute coronary syndrome of left dominant coronary anatomy. Early adequate medical and intervention treatment has been shown to reduce cardiac events of these high risk group patients. But further study should be carried out involving large number of population covering estimated sample size on the same topic.

\section{Conflict of Interest - None.}

\section{References:}

1. Zachariah JP, Vasan RS and D'Agostino RB. The Burden of Increasing Worldwide Cardiovascular Disease. In: V Fuster, R A Walsh, and RA Harrington. Eds. Hurst's The Heart. 13th ed. New York: The McGraw-Hill Companies, Inc 2011;1:17-42.

2. Wong ND. Epidemiological studies of coronary heart disease and the evolution of preventive cardiology. Nature Reviews Cardiology 2014;11(5): 276-289.

3. Islam AKMM and Majumder AAS. Coronary artery disease in Bangladesh: A review. Indian Heart Journal 2013; 65(4): 424-435.

4. Mauri L and Bhatt DL. Percutaneous Coronary Intervention. In: D L Mann, D PZipes, P Libby, and R OBonow. Eds. Braunwald's Heart Disease - A Textbook of Cardiovascular Medicine. 10th ed. Philadelphia: Elsevier Saunders 2015;1245-1268.

5. Banning AP, Baumbach A, Blackman D, Curzen N, Devadathan S, Fraser D, et al. Percutaneous coronary intervention in the UK: recommendations for good practice. Brit Med J 2015; 101(Suppl 3): 1-13.

6. Kuno T, Numasawa Y, Miyata H, Takahashi T, Sueyoshi $\mathrm{K}$, Ohki T, et al. Impact of Coronary Dominance on InHospital Outcomes after Percutaneous Coronary 
Intervention in Patients with Acute Coronary Syndrome. PLoS ONE 2013; 8(8): e72672-72674.

7. Parikh NI, Honeycutt EF, Roe MT, Neely M, Rosenthal EJ, Mittleman MA, et al. Left and Codominant Coronary Artery Circulations Are Associated with Higher In-Hospital Mortality Among Patients Undergoing Percutaneous Coronary Intervention for Acute Coronary Syndromes. Report from the National Cardiovascular Database Cath Percutaneous Coronary Intervention (Cath PCI) Registry. Circulation: Cardiovascular Quality and Outcomes 2012; 5(6): 775-782.
8. Akanda MAK, Ali SY, Islam AEMM, Rahman MM, Parveen A, Kabir MK, et al. Demographic Profile, Clinical Presentation \& Angiographic Findings in 637 Patients with Coronary Heart Disease. Faridpur Medical College Journal 2011; 6(2): 82-85.

9. Ullah M, Khalequzzaman M, Habib SMA, Kar N and Islam MN. Angiographic correlation of ST segment depression on admission ECG in patient with ACS. Bangladesh Heart Journal, 2006; 21(2);72-77.

10. Moyeen U. Association of central pulse pressure with the extent of angiographically etected coronary artery disease. MD. University of Dhaka 2011; 35. 